\title{
Escala de Satisfação no Domínio Académico em Universitários Portugueses
}

\author{
Academic Domain Satisfaction Scale in Portuguese College Students
}

\author{
Leandro S. Almeida ${ }^{1}$, Maria do Céu Taveira ${ }^{2}$, Francisco Peixoto ${ }^{3}$, José Castro Silva ${ }^{4}$ \\ e Maria João Gouveia ${ }^{5}$
}

\begin{abstract}
Resumo
A satisfação dos estudantes tem sido valorizada como um indicador da qualidade das instituições de Ensino Superior, e como variável relevante na explicação do envolvimento, aprendizagem e persistência dos estudantes. A satisfação, associada aos diversos contextos da experiência académica, pode ser entendida como a apreciação que os estudantes realizam da instituição, clima académico, curso e relações interpessoais. Com base numa amostra de 704 estudantes, de duas instituições portuguesas, uma pública e outra privada, analisam-se as evidências de validade da Escala de Satisfação no Domínio Académico. Os resultados da análise fatorial confirmatória sugerem a natureza unidimensional da escala. Por outro lado, níveis adequados de consistência interna dos seus itens, assim como correlações significativas entre o resultado na escala e indicadores da disposição afetiva, rendimento, e intenção de abandono escolar, apontam no sentido da validade da medida.
\end{abstract}

Palavras-chave: ensino superior, satisfação académica, sucesso académico

\begin{abstract}
Students' satisfaction has been valued as an indicator of the quality of higher education institutions, and as a relevant variable in explaining their involvement, learning and persistence. Satisfaction, associated to the diverse contexts of students' academic experience, is understood as a construct of students' evaluation of the institution, academic climate, course and interpersonal relationships. Based on a sample of students from two Portuguese Higher Education institutions, one public and another private, we analyze the validity evidence of the Academic Domain Satisfaction Scale. The results of a confirmatory factorial analysis suggest the onedimensional nature of the scale. On the other hand, adequate levels of internal consistency of its items, as well as significant correlations between the result in the scale and indicators of the students' affective disposition, academic performance and dropout intentions, point towards the validity of the measure.
\end{abstract}

Keywords: higher education, academic satisfaction, academic success

Agradecimentos: A presente investigação foi financiada pela Fundação para a Ciência e Tecnologia através do financiamento ao CIE-ISPA (UID/CED/04853/2013 e UID/CED/04853/2016) e ao CIEd-UMinho (UID/CED/01661/2019).

\footnotetext{
${ }^{1}$ Doutorado. Professor Catedrático. Instituto de Educação, Universidade do Minho, Campus de Gualtar 4710-057 Braga, Portugal. Tel.: +35125360424. E-mail: leandro@ie.uminho.pt

${ }^{2}$ Doutorada, Professora Auxiliar, Escola de Psicologia, Universidade do Minho, Campus de Gualtar 4710-057 Braga, Portugal. Tel.: +351253604671. E-mail: ceuta@psi.uminho.pt

3 Doutorado. Professor Associado. ISPA - Instituto Universitário, CIE-ISPA, Rua Jardim do Tabaco, n³4, 1149-041 Lisboa, Portugal. Tel.: +351218811700. E-mail: fpeixoto@ispa.pt

${ }^{4}$ Doutorado. Professor Auxiliar. ISPA - Instituto Universitário, CIE-ISPA, Rua Jardim do Tabaco, nº34, 1149-041 Lisboa, Portugal. Tel.: +351218811700. E-mail: jcsilva@ispa.pt

5 Doutorada. Professora Auxiliar. ISPA - Instituto Universitário, APPsyCI, Rua Jardim do Tabaco, n³4 1149-041 Lisboa, Portugal. Tel.: +351218811700. E-mail: mjgouveia@ispa.pt

Revista Iberoamericana de Diagnóstico y Evaluación - e Avaliação Psicológica. RIDEP · N54 · Vol.1 · 93-101 · 2020 ISSN: 1135-3848 print /2183-6051online
} 


\section{Introdução}

A experiência académica dos estudantes do Ensino Superior é comumente marcada por um conjunto diversificado de perceções que moldam o seu grau de satisfação. Essa experiência académica envolve o acesso e utilização dos espaços, equipamentos e recursos da instituição, a aprendizagem e rendimento académico, a organização do curso e ensino dos professores, as relações com os colegas e os serviços de apoio (Astin, 1993; Casanova, Esteban, Cervero, Bernardo, \& Almeida, 2017; Fernández, Araújo, Vacas, Almeida, \& Gonzalez, 2017; Grebennikov \& Shah, 2013; Pinto, Faria, Pinto, \& Taveira, 2016). A satisfação é determinada por variáveis relativas à instituição, curso, relações interpessoais, perspetiva de carreira e caraterísticas pessoais (Hu \& Kuh, 2002; Pinto et al., 2016), nomeadamente quando as expectativas iniciais ou as necessidades dos estudantes se concretizam, face à realidade académica (Lent et al., 2005; Lent, Taveira, Sheu, \& Singley, 2009; Pinto, Quadros, Cruz, \& Conrad, 2017; Soares et al., 2018; Souza \& Reinert, 2010; Tomás \& Gutiérrez, 2019). A satisfação representa, assim, um indicador da avaliação feita pelos estudantes da sua experiência de bem-estar subjetivo no domínio académico, afetando a qualidade dessa mesma experiência, em termos de frequência e de intensidade (Novo, 2005; Wilkins, Butt, Kratochvil, \& Balakrishnan, 2016).

Pela sua relevância, a satisfação académica é algumas vezes assumida como um indicador de sucesso e da qualidade das instituições de Ensino Superior. Em Portugal, uma menor satisfação académica dos estudantes do primeiro ano, pode decorrer do número expressivo de estudantes não colocados na sua primeira opção do par curso/instituição, fruto do sistema de acesso ao ensino superior (e.g., numerus clausus de vagas, preenchido através de uma nota de acesso que considera a classificação final no ensino secundário e em provas específicas de acesso) (Fonseca, Dias, Sá, \& Amaral, 2013). Esta insatisfação com o curso frequentado pode ter maior expressão em Portugal, dado tratarem-se de cursos de banda estreita, conducentes a formações científico-técnicas e a atividades profissionais específicas, caraterística de cursos que a investigação tem demonstrado registarem bastante abandono associado (e.g., Suhre, Jansen, \& Harskamp, 2007).

Pela sua abrangência, antecipa-se a relevância da satisfação académica para o bem-estar, formação e sucesso académico dos estudantes do ensino superior. Em estudantes do primeiro ano, a satisfação aparece associada à persistência ou abandono do curso e/ou instituição, assumindo um papel relevante na adaptação ao ensino superior (Arantes, Pátaro, Pinheiro, \& Gonçalo, 2018; Teixeira, Dias, Wottrich, \& Oliveira, 2008; Zannon, 2011). Sheu e Bordon (2017), por sua vez, referem que aspetos como a autoeficácia académica, a perceção de avanço nos objetivos escolares e a perceção de apoio por parte de docentes, colegas e familiares, a par de uma disposição afetiva positiva e conscienciosidade, afetam positivamente a satisfação com o domínio de vida académica, e que esta é um preditor da satisfação geral com a vida. De igual modo, a qualidade das relações interpessoais com colegas, funcionários e professores, contribui para os índices de satisfação dos estudantes (e.g., Veras \& Ferreira, 2010).

Pela pluralidade de aspetos considerados, a avaliação da satisfação académica recorre frequentemente a escalas multidimensionais. A título de exemplo, a relação com os colegas e professores (dimensão social), o bem-estar e a autoconfiança (dimensão pessoal), as infraestruturas, equipamentos e serviços (dimensão institucional), e a estrutura curricular do curso e relevância da formação auferida para os projetos vocacionais (dimensão curso), ilustram algumas das dimensões consideradas nos instrumentos usados na avaliação da satisfação nas diversas áreas da vida académica (e.g., Araújo, Almeida, Guisande, \& Paul, 2006; Osti \& Almeida, 2019). Estas dimensões convergem para um fator geral de segunda ordem que pode designar-se, genericamente, de satisfação académica, havendo ainda alguns autores que apontam as dimensões institucional e curso como as mais determinantes da satisfação académica dos estudantes.

A investigação na área sugere, além disso, que a satisfação académica dos estudantes se diferencia segundo o sexo, com as raparigas a apresentarem níveis mais elevados de satisfação e, 
também, de rendimento académico (Belvis, Moreno, \& Ferrán, 2009; Grau \& SanchoAlvarez, 2011). Por outro lado, um maior número de estudos aponta para uma correlação positiva entre níveis de satisfação e rendimento académico (Monteiro, Tavares, \& Pereira, 2008; Morton, Mergler, \& Boman, 2014; Schleich et al., 2006; Souza \& Reinert, 2010). Os resultados destes estudos indicam que estudantes mais satisfeitos são mais resilientes, entusiastas e implicados nas aprendizagens e atividades do curso. Tais vivências elevam os níveis de adaptação à instituição e ao curso (Araújo, 2017; Casanova, Esteban, Cervero, Bernardo, \& Almeida, 2017; Ferrão \& Almeida, 2018). Efetivamente, níveis mais elevados de satisfação aparecem associados a maior envolvimento em atividades significativas no campus, em particular, oportunidades de estabelecimento de relações sociais estáveis e de participação nas atividades de sala de aula, bem como realização de trabalhos ou integração em pesquisas dos docentes (e.g., Figuera \& Torrado, 2015; Yu \& Wright, 2016). Neste sentido, o conhecimento dos níveis de satisfação académica dos estudantes permite, às instituições de ensino superior, implementar medidas que ajudem os estudantes a ultrapassar dificuldades na sua adaptação e sucesso académicos.

Dada a relevância da satisfação académica para a definição de políticas institucionais que favoreçam a permanência, sucesso e conclusão dos cursos, pelos estudantes, apresentamos neste artigo evidências de validade de uma escala de screening que pode ser usada junto de amostras alargadas de estudantes, para sinalizar situações de experiência de (in)satisfação académica. Neste sentido, a par da testagem da estrutura dimensional da escala, unidimensional, analisa-se a consistência interna dos seus itens e a correlação dos resultados na escala com critérios externos, recorrendo a medidas de autorrelato e, também, a medidas objetivas do rendimento escolar dos estudantes.

\section{Método}

\section{Participantes}

Participaram 704 estudantes universitários de ambos os sexos, maioritariamente do sexo feminino $(78.7 \%)$ e com idades compreendidas entre 18 e 59 anos $(M=20.74, D P=5.01)$, a frequentarem diferentes cursos, a maioria, nas áreas do Direito, Ciências Sociais e Serviços $(\mathrm{n}=363.61 \%)$, Arquitetura, Artes Plásticas e Design ( $\mathrm{n}=107,18 \%)$, Ciências da Educação $(\mathrm{n}=96.16 \%)$, e Tecnologias $(\mathrm{n}=26.4 \%)$. A quase totalidade dos estudantes $(98.4 \%)$ frequenta o $1^{\circ}$ ano dos respetivos cursos.

\section{Instrumentos}

Escala de Satisfação no Domínio Académico (Lent, Singley et al., 2005). Utilizou-se a versão portuguesa da escala (Lent et al., 2009), constituída por sete itens (e.g., "Gosto do nível de estimulação intelectual do meu curso"), de resposta numa escala de tipo Likert, que requer que o sujeito indique o seu nível de satisfação com diferentes aspetos da experiência de vida académica. No presente estudo, a versão da medida utilizada incluiu uma escala de resposta com seis pontos, em que zero significa discordo totalmente e cinco significa concordo totalmente. A escala apresentou valores de consistência adequados com amostras norte-americanas $(\alpha=.86$ a .87) (Lent et al., 2005; Singley et al., 2010) e do noroeste português $(\alpha$ teste re-teste $=.85$ e .89 ) (Lent et al., 2009, Lent et al., 2012). No contexto americano, a escala registou correlações positivas com medidas de afeto positivo, satisfação no domínio social e satisfação geral com a vida (Lent et al., 2005; Singley et al., 2010). No contexto português, registaram-se correlações positivas com medidas de afeto positivo e satisfação geral com a vida (Lent et al., 2009, Lent et al., 2012).

Escalas do Afeto Positivo e do Afeto Negativo (Wosnitza, Karabenick, Peixoto, \& Nolden, 2018). Estas escalas foram usadas para avaliar a tendência para experimentar emoções positivas ou negativas. Incluem um total de oito itens, quatro itens que avaliam afetos positivos (e.g., "Quando penso na minha vida na universidade, sinto-me feliz") e quatro itens que avaliam afetos negativos (e.g., "Quando penso na minha vida na universidade, sinto-me angustiado/a"), face aos quais os participantes avaliam a frequência, numa escala de 1 (muito pouco ou nada) a 5 (extremamente). Estas variáveis de personalidade podem moldar a forma como os indivíduos lidam com as tarefas e ambiente académico (e.g., Sheu \& Bordon, 2017). No presente estudo, a versão da 
medida utilizada incluiu uma escala de resposta com seis pontos, em que zero significa muito pouco ou nada e cinco significa extremamente.

$\mathrm{O}$ rendimento académico foi avaliado tendo por base a média das notas das unidades curriculares realizadas pelos estudantes, no segundo semestre.

A intenção de abandono dos estudos foi avaliada a partir da resposta a dois itens que apontavam para a ideia de desistir do curso (e.g. "Estou seriamente a pensar abandonar os meus estudos"), numa escala de zero (Discordo Totalmente) até seis (Concordo Totalmente) pontos.

\section{Procedimentos}

O estudo decorreu após recebidas autorizações dos responsáveis pelas instituições e cursos envolvidos na investigação. Os dados foram recolhidos em contexto de sala de aula, no âmbito de um estudo mais vasto sobre os fatores de abandono escolar no Ensino Superior. Os estudantes foram informados dos objetivos do estudo e deram o seu consentimento informado, prévio à aplicação das escalas.

\section{Análise de Dados}

A análise das propriedades psicométricas da escala foi realizada do ponto de vista da validade interna, da consistência interna e da validade de constructo. Para a análise da validade interna da escala, recorreu-se a uma análise fatorial confirmatória (AFC), utilizando o estimador de máxima verosimilhança robusto (MLR), disponível no MPlus (v. 7.4., Muthén \& Muthén, 1998-2016, CA). A adequação do modelo aos dados foi avaliada através do Comparative Fit Index (CFI), Tucker-Lewis Index (TLI), Root Mean Square Error of Approximation (RMSEA) e Standardized Root Mean Square Residual (SRMR), uma vez que, devido à dimensão da amostra, o valor do qui-quadrado indicaria sempre desajustamento do modelo (Kline, 2011; Shumacker \& Lomax, 2004). Para o CFI e o TLI, consideraram-se aceitáveis, valores acima de .95 , enquanto para o RMSEA e o SRMR, consideraram-se adequados, os valores abaixo de .06 (Browne \& Cudeck, 1993; Schmitt, 2011). A consistência interna foi analisada através do cálculo do alfa de Cronbach, devido à sua popularidade (e.g., Taber, 2018) e, também, do ómega de McDonald, devido à sua maior robustez (Geldhof, Preacher, \& Zyphur, 2014). Para a validade de critério, correlacionaram-se os resultados na escala de satisfação com os valores na escala de emoções positivas e negativas, com o rendimento académico e com a intenção de abandono dos estudos.

\section{Resultados}

Centrado na validade da escala, este artigo descreve a sua estrutura ou dimensionalidade (validade interna), a consistência ou homogeneidade dos seus itens e a validade de critério ou externa dos resultados. Em termos de dimensionalidade, a proposta da versão original da escala do autor é que os sete itens convergem para uma única dimensão ou fator, entendido como satisfação académica dos estudantes. Nesse sentido, procedemos a uma análise fatorial confirmatória e os resultados indicam, globalmente, um ajustamento aceitável do modelo aos dados $\left(\chi^{2}(14)=130.74, \quad p<.001, \quad\right.$ CFI $=.94$, TLI=.91, RMSEA $=.111 \quad[0.094, \quad 0.128]$, SRMR=.036). Esta apreciação é feita apesar do valor elevado de RMSEA, já que este valor pode ser justificado pelo facto de este índice ser penalizado em modelos com um número diminuto de variáveis e graus de liberdade (Kenny, Kaniskan, \& McCoach, 2015). A análise dos índices de modificação sugere uma melhoria da adequação do modelo aos dados, correlacionando o item 1 com o item 2 e o item 6 com o item 7. As correlações entre estes pares de itens, traduzindo variância comum não captada pelo fator geral, pode estar associado, no caso do Item 1 "Sinto-me satisfeito(a) com a decisão de me formar na área pretendida" e item 2 "Estou confortável com o ambiente educacional do meu curso", à satisfação dos estudantes com a implementação da sua escolha e adaptação ao curso. Ou seja, ambos os itens expressam alguma correspondência entre a decisão da escolha e expectativas inerentes e adaptação académica experienciada. Por sua vez, a correlação entre o item 6, "Sinto-me entusiasmado(a) com as matérias do curso escolhido", e o item 7, "Gosto do quanto tenho aprendido nas aulas", parece decorrer do próprio 
conteúdo comum aos dois itens, ou seja, a satisfação com os conteúdos curriculares ou as aprendizagens específicas do curso frequentado.

Feitas estas duas alterações, o modelo revela um bom ajustamento aos dados $\left(\chi^{2}(12)=59.15\right.$, $p<.001, \quad$ SRMR $=.023, \quad$ CFI $=.98, \quad$ TLI $=.96$, RMSEA $=.076 \quad[.057$, .096]). Na Figura 1 apresentam-se os resultados finais da análise fatorial confirmatória da escala de satisfação no domínio académico. Observa-se que os valores de saturação dos itens são bastante elevados, variando entre .69 (item 1) e .91 (item 3).

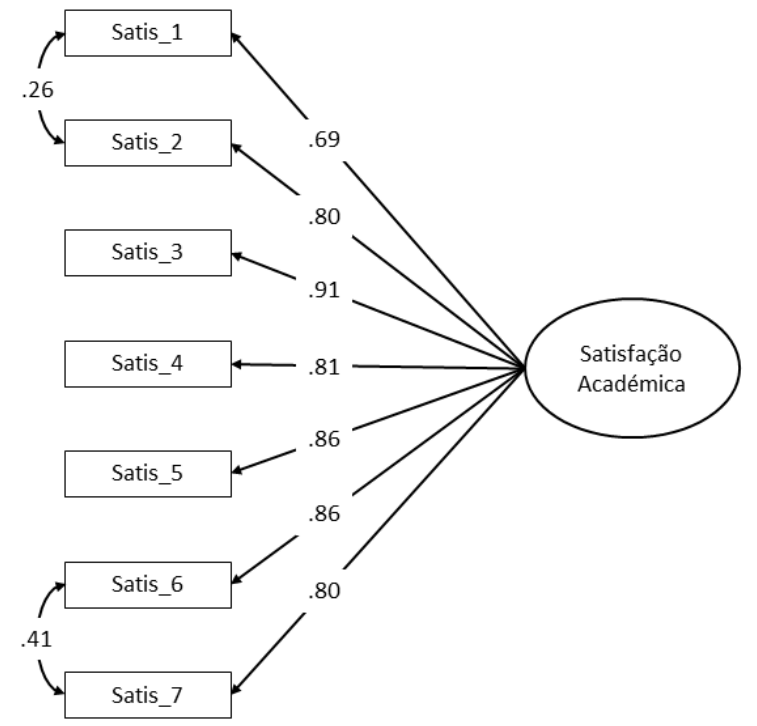

Figura 1. Análise Fatorial Confirmatória da

Escala de Satisfação no Domínio Académico

Quadro 1. Correlação item-total corrigida, para os itens da escala de Satisfação no Domínio

Académico

\begin{tabular}{lc}
\hline & $\begin{array}{c}\text { Correlação } \\
\text { item-total } \\
\text { corrigida }\end{array}$ \\
\hline $\begin{array}{l}\text { 1. Sinto-me satisfeito(a) com a decisão de } \\
\text { me formar na área pretendida. }\end{array}$ & .69 \\
$\begin{array}{l}\text { 2. Estou confortável com o ambiente } \\
\text { educacional do meu curso. }\end{array}$ & .79 \\
$\begin{array}{l}\text { 3. Na maior parte das vez, estou a gostar do } \\
\text { meu curso. } \\
\text { 4. De um modo geral, estou satisfeito(a) }\end{array}$ & .89 \\
com a minha vida académica. & .78 \\
$\begin{array}{l}\text { 5. Gosto do nível de estimulação intelectual } \\
\text { do meu curso. }\end{array}$ & .83 \\
6. Sinto-me entusiasmado(a) com as \\
matérias do curso escolhido.
\end{tabular}

A consistência interna da escala revela valores muito elevados, quer para o alfa de Cronbach $(\alpha=.938)$, quer para o ómega de McDonald
$(\Omega=.939)$. No Quadro 1, apresentam-se os resultados da correlação item-total corrigida, para os itens da escala de satisfação no domínio académico. Nesta análise, os valores da correlação entre cada um dos itens e o score global são também bastante elevados, variando entre .69 (item 1) e .89 (item 3). O item 1, pelo facto de ser o primeiro item da escala, pode suscitar alguma especificidade nas respostas dos estudantes, refletindo a adaptação dos estudantes ao conteúdo da escala.

No Quadro 2, apresentam-se os resultados da análise das correlações do score global da satisfação académica com as emoções positivas, as emoções negativas, o rendimento académico, e a intenção de abandono. Os resultados obtidos estiveram de acordo com o esperado. Assim, a medida de satisfação no domínio académico correlacionou-se positiva e significativamente com o rendimento académico e com as emoções positivas e, negativamente, com as emoções negativas e a intenção de desistir do curso. De referir a reduzida correlação, ainda que estatisticamente significativa, encontrada entre a medida objetiva do rendimento académico e os níveis de satisfação dos estudantes.

Quadro 2. Correlações da escala de Satisfação no Domínio Académico com outras medidas

\begin{tabular}{lc}
\hline & Satisfação Académica \\
\hline Emoções Positivas & $.66 * * *$ \\
Emoções Negativas & $-.33 * * *$ \\
Rendimento Académico & $.13 * *$ \\
Intenção de desistir do curso & $-.40 * * *$ \\
\hline Nota. ${ }^{* *} p<.01, * * * p<.001$ &
\end{tabular}

\section{Discussão}

A satisfação académica tem sido considerada um indicador de adaptação e bem-estar em ambientes de trabalho escolar, com uma relação positiva com a satisfação geral com a vida (e.g., Osti \& Almeida, 2019; Sheu \& Bordon, 2017). A satisfação com a vida académica reflete o processo de correspondência entre as expectativas, motivação e envolvimento dos estudantes e as expectativas e resposta das instituições de ensino em relação aos mesmos (Arantes et al., 2018; Fernandez et al., 2017; Zannon, 2011). Por esta razão, a satisfação académica pode refletir, em certa medida, a qualidade do ensino superior 
(Souza \& Reinert, 2010), e as necessidades de apoio por parte dos estudantes (Pinto et al., 2016).

Face à necessidade de avaliação deste constructo, o presente estudo teve como objetivo analisar a validade e a precisão da versão portuguesa da escala de satisfação no domínio académico, em estudantes portugueses do ensino superior. Em linha com estudos prévios sobre o funcionamento da escala (Lent et al., 2005; Lent et al., 2009; Lent et al., 2012; Singley et al., 2010), obtiveram-se bons indicadores de validade e precisão dos resultados neste estudo. A partir dos resultados da análise fatorial confirmatória, observou-se que o modelo teórico unidimensional da escala, com sete itens, apresenta índices adequados de ajustamento, registando-se índices elevados de saturação dos itens no fator geral obtido. O elevado coeficiente de consistência interna e as correlações de cada item com a pontuação total na escala demonstram a sua precisão e valida aplicações prévias da escala (Lent et al., 2009; Lent et al., 2012). Finalmente, as correlações obtidas com a medida dos afetos positivos e afetos negativos, a correlação negativa com a intenção de desistência do curso e a relação positiva, ainda que baixa, com o rendimento académico vão no sentido da validade de critério da escala. Estes valores sugerem que podemos encontrar níveis maiores de satisfação académica nos estudantes que apresentam pontuações mais elevadas nos afetos positivos, pontuando inversamente nos afetos negativos e na intenção de desistir do curso, como se registou em outros estudos (Arantes et al., 2018; Lima-Castro et al., 2019; Morton et al., 2014; Souza \& Reinert, 2010; Teixeira et al., 2008). A baixa correlação verificada entre os níveis de satisfação e a medida objetiva do rendimento académico dos estudantes pode refletir a natureza mais abrangente da satisfação académica, por exemplo as vivências e a avaliação feita do clima e ambiente académicos. Aliás, é possível que estudantes com um mesmo nível de rendimento experienciem níveis diferenciados de satisfação em função dos objetivos pessoais. Por outro lado, a baixa correlação encontrada pode refletir especificidades curriculares nos conteúdos, dinâmica das aulas e formas de avaliação de curso para curso, prejudicando o sentido linear das correlações estimadas.
A finalizar, algumas limitações merecem ser superadas em futuras investigações. Pela relevância do constructo, torna-se importante recolher dados com esta escala em amostras mais representativas dos estudantes portugueses, do sector público e privado, do ensino universitário e politécnico. A par de alunos colocados e não colocados em cursos/instituições de primeira opção, justificar-se-ia conhecer a distribuição dos resultados neste constructo, considerando públicos não tradicionais, como por exemplo, estudantes mais velhos. Por último, estudos de validade deveriam incluir a aplicação de escalas com constructos psicológicos mais próximos, como a autoeficácia académica e a satisfação com a vida em geral, assim como indicadores comportamentais retidos do quotidiano do estudante, como presença às aulas, atividades de envolvimento no campus e estilos de vida. Igualmente importaria aprofundar a baixa correlação encontrada entre níveis de satisfação e rendimento académico dos estudantes, por exemplo, ponderando as especificidades das áreas científicas dos cursos e introduzindo possíveis variáveis moderadoras naquela relação.

\section{Referências}

Arantes, V. A., Pátaro, C. S. O., Pinheiro, V. P. G., \& Gonçalo, M. F. (2018). Felicidade e bem-estar da juventude brasileira. Notandum, 46, 55-68. doi:10.4025/notandum.46.4

Araújo, A. M. (2017). Sucesso no Ensino Superior: Uma revisão e conceptualização. Revista de Estudios $e$ Investigación en Psicología y Educación, 4, 132-141. doi:10.17979/reipe.2017.4.2.3207

Araújo, B. R., Almeida, L. S., Guisande, M. A., \& Paul, M. C. (2006). Vivências e satisfação académicas em estudantes do curso de Enfermagem. Revista Galego-Portuguesa de Psicoloxía e Educación, 13, 363-371.

Astin, A. (1993). What matters in college? Four critical years revisited. San Francisco, CA: Jossey-Bass.

Belvis, E., Moreno, M. V., \& Ferrer, F. (2009). Los factores explicativos del éxito y fracaso académico en las universidades españolas, en los años del cambio hacia la convergencia 
europea. Revista Española de Educación Comparada, 15, 61-92.

Browne, M. W., \& Cudeck, R. (1993). Alternative ways of assessing model fit. In K. A. Bollen \& J. S. Long (Eds.), Testing structural equation models (pp. 136-162). Newbury Park, California: Sage.

Casanova, J. R., Esteban, M., Cervero, A., Bernardo, A., \& Almeida, L. S. (2017). Questões vocacionais e de aprendizagem no abandono precoce do Ensino Superior. In A. P. Marques, C. Sá, J. R. Casanova \& L. S. Almeida (Orgs.), Ser diplomado do Ensino Superior: Escolhas, percursos e retornos (pp. 175-190). Braga: Centro de Investigação em Educação (CIEd), Instituto de Educação, Universidade do Minho.

Direção-Geral do Ensino Superior (2018). Índice por área de estudo e curso._Acedido em 7 de Março de 2019. www.dges.gov.pt/guias/indarea.asp?area=II

Fernández, M. F. P., Araújo, A. M., Vacas, C. T., Almeida, L. S., \& Gonzalez, M. S. R. (2017). Predictors of students' adjustment during transition to university in Spain. Psicothema, 29, 67-72. doi:10.7334/psicothema2016.40

Ferrão, M. E., \& Almeida, L. S. (2018). Multilevel modeling of persistence in Higher Education. Ensaio: Avaliação e Politicas Públicas em Educação, 26, 664-683. doi:10.1590/S0104-40362018002601610

Figuera, P., \& Torrado, M. (2015). The transition to university of at-risk groups in Spain: The case of students from vocational education and training. Revista de Cercetare si Interventie Sociala, 49, 23-40.

Fonseca, M., Dias, D., Sá, C., \& Amaral, A. (2013). Waves of (dis)satisfaction: Effects of the numerus clausus system in Portugal. European Journal of Education, 49, 144-158. doi:10.1111/ejed.12042

Geldhof, G. J., Preacher, K. J., \& Zyphur, M. J. (2014). Reliability estimation in a multilevel confirmatory factor analysis framework. Psychological Methods, 19, 72-91. doi:10.1037/a0032138

Grau, R., \& Sancho-Alvarez, C. (2011). Posibles causas del fracaso escolar y el retorno al sistema educativo. Hekademos. Revista Digital de Educación, 4(9), 55-76.
Grebennikov, L., \& Shah, M. (2013). Monitoring trends in student satisfaction. Tertiary Education in Management, 19, 301-322. doi:10.1080/13583883.2013.804114

Hu, S., \& Kuh, G. D. (2002). Being (dis)engaged in educationally purposeful activities: The influences of student and institutional characteristics. Research in Higher Education, 43, 555-575. doi:10.1023/A:102011423

Kenny, D. A., Kaniskan, B., \& McCoach, D. B. (2015) The performance of RMSEA in models with small degrees of freedom. Sociological Methods and Research, 44, 486507. doi:10.1177/0049124114543236

Kline, R. B. (2011). Principles and practice of structural equation modeling (3rd Ed.). New York: Guilford Press.

Lent, R. W., Singley, D., Sheu, H., Gainor, K. A., Brenner, B. R., Treistman, D., et al. (2005). Social cognitive predictors of domain and life satisfaction: Exploring the theoretical precursors of subjective well-being. Journal of Counseling Psychology, 52, 429-442. doi:10.1177/1069072709354199

Lent, R. W., Taveira, M., \& Lobo, C. (2012). Two tests of the social cognitive model of wellbeing in Portuguese college students. Journal of Vocational Behavior, 80, 362-371. doi:10.1016/j.jvb.2011.08.009

Lent, R. W., Taveira, M., Sheu, H., \& Singley, D. (2009). Testing a social cognitive model of college adjustment in Portugal. Journal of Vocational Behavior, 74, 190-198. doi:10.1016/j.jvb.2008.12.006

Lent, R. W., \& Brown, S. D. (2017). Social cognitive career theory in a diverse world: Guest Editors' Introduction. Journal of Career Assessment, 25, 3-5. doi:10.1177/1069072716657811

Lima-Castro, S., Peña-Contreras, E., AguilarSizer, M., Pacheco, A. B., \& Arias-Medina, P. (2019). Aproximación a un Modelo de Bienestar en Adultos Ecuatorianos. Revista Iberoamericana de Diagnóstico y Evaluación - e Avaliação Psicológica, 51, 5-18. doi.10.21865/RIDEP51.2.01

Monteiro, S. O. M., Tavares, J. P. C., \& Pereira, A. M. C. (2008). Optimismo disposicional em estudantes. Estudos de Psicologia, 13(1), 2329. doi:10.1590/S1413-294X2008000100003 
Morton, S., Mergler, A., \& Boman, P. (2013). Managing the transition: The role of optimism and self-efficacy for first-year Australian university students. Australian Journal of Guidance and Counselling, 24, 90-108. doi:10.1017/jgc.2013.29.

Muthén, L. K., \& Muthén, B. O. (1998-2016). Mplus user's guide (7th ed.). Los Angeles, CA: Author.

Novo, R. F. (2005). Bem-estar e psicologia: Conceitos e propostas de avaliação. Revista Iberoamericana de Diagnóstico y Evaluación - e Avaliação Psicológica, 20(2), 183-203. Retrieved from http://hdl.handle.net/10451/17844

Osti, A., \& Almeida, L. S. (2019). Satisfação académica no Ensino Superior. In Leandro S. Almeida (Ed.), Estudantes do Ensino Superior: Desafios e oportunidades (pp. 99114). Braga: ADIPSIEDUC.

Pinto, J. C., Faria, L., Pinto, H. R., \& Taveira, M.C. (2016). Identificação de necessidades de intervenção psicológica: Um estudo-piloto no ensino superior português. Psicologia USP, 3, 459-472. doi:10.1590/0103-656420150015

Pinto, N. G. M., Quadros, R. C., Cruz, F. V., \& Conrad, C. C. (2017). Satisfação acadêmica no Ensino Superior brasileiro: Uma análise das evidências empíricas. Revista Brasileira de Ensino Superior, Passo Fundo, 3, 3-17. doi:10.18256/2447-3944.2017.v3i2.1600

Schleich, A. L. R., Polydoro, S. A. J., \& Santos, A. A. A. (2006). Escala de satisfação com a experiência acadêmica de estudantes de ensino superior. Avaliação Psicológica, 5(1), 11-20.

Schmitt, T. A. (2011). Current methodological considerations in exploratory and confirmatory factor analysis. Journal of Psychoeducational Assessment, 29, 304-321. doi:10.1177/0734282911406653

Singley, D., Lent, R. W., \& Sheu, H. (2010). Longitudinal test of a social cognitive model of academic and life satisfaction. Journal of Career Assessment, 18, 133-146. doi:10.1177/1069072709354199

Sheu, H., \& Bordon, J. J. (2017). SCCT Research in the International context: Empirical evidence, future directions, and practical implications. Journal of Career Assessment, 25, 58-74. doi:10.1177/1069072716657826

Shumacker, R. E., \& Lomax, R. G. (2004). A beginner's guide to structural equation modeling (2nd Ed.). Mahwah, NJ: Lawrence Erlbaum.

Soares, A. B., Leme, V. B. R., Gomes, G., Penha, A. P., Maia, F. A., Lima, C. A. et al. (2018). Expectativas acadêmicas de estudantes nos primeiros anos do Ensino Superior. Arquivos Brasileiros de Psicologia, 70(1), 206-223.

Souza, S. A., \& Reinert, J. N. (2010). Avaliação de um curso de ensino superior através da satisfação/insatisfação discente. Avaliação (Campinas), Sorocaba,15, 159-176. doi:10.1590/S1414-40772010000100009

Suhre, C., Jansen, E. P. W. A., \& Harskamp, E. (2007). Impact of degree program satisfaction on the persistence of college students. Higher Education, 54, 207-226. doi:10.1007/s10734-005-2376-5.

Taber, K. S. (2018). The use of Cronbach's alpha when developing and reporting research Instruments in science education. Research in Science Education, 48, 1273. doi:10.1007/s11165-016-9602-2

Teixeira, M. A. P., Dias, A. C. G., Wottrich, S. H., \& Oliveira, A. M. (2008). Adaptação à universidade em jovens calouros. Psicologia Escolar e Educacional, 12, 185-202. doi:10.1590/S1413-85572008000100013

Tomás, J. M., \& Gutiérrez, M. (2019). Aportaciones de la teoría de la autodeterminación a la predicción de la satisfacción académica en estudiantes universitarios. Revista de Investigación Educativa, $\quad 37, \quad 471-485$. doi:10.6018/rie.37.2.328191

Veras, R. S., \& Ferreira, S. P. A. (2010). The affection in the teacher-student relationship and its implications on learning in the university context. Educar em Revista, 38, 219235. doi:10.15:90/S0104-40602010000300015.

Wilkins, S., Butt, M. M., Kratochvil, D., \& Balakrishnan, M. S. (2016). The effects of social identification and organizational identification on student commitment, achievement and satisfaction in higher education. Studies in Higher Education, 
41(12),

2232-2252.

doi:10.1080/03075079.2015.1034258

Wosnitza, M., Karabenick, S. A., Peixoto, F., \& Nolden, P. (2018). Scales of Positive and Negative Emotions. RWTH. Aachen University: Aachen.

Yu, B., \& Wright, E. (2016). Socio-cultural adaptation, academic adaptation and satisfaction of international higher degree research students in Australia. Tertiary Education and Management, 22, 49-64. doi:10.1080/13583883.2015.1127405

Zanon, C. (2011). Construção de uma escala de afetos e suas relações com os cinco grande fatores de personalidade (Big Five). Tesis Doctoral. Universidade Federal do Rio Grande do Sul, Instituto de Psicologia. 